\title{
Comment on "ATP bioluminescence - for kitchen hygiene but also cleaning control of surgical instruments"
}

\author{
Peter Hoffman \\ Laboratory of Healthcare-associated Infection, Health Protection Agency, UK \\ doi:10.3396/ijic.V4i1.011.08
}

This paper addresses one of the most difficult areas of infection control -how to assure the quality of processes that rely mainly on effective human procedures. There are two main approaches to this:

- Product control - in which a product is assessed for suitability after it has been processed

- Process control - in which the parameters of a successful process are established and those parameters are monitored rather than the endproduct.

The paper by Hansen, Hilgenhöner, and Popp is an example of product control; assessing the cleanliness of an endoscope after cleaning. It happens to use an ATP-based method as these are established and available but other methods are well discussed in that paper. This commentary is not about which methods can be used to assess the cleanliness of a product but if and when this approach is appropriate for use.

The main criticism of this approach when applied to endoscopes is summed-up by an appreciation that the easiest places on an endoscope to sample are also the easiest places to clean and, conversely, the most difficult places to clean are also the most difficult places to sample. With endoscopes, the most difficult places for both cleaning and sampling are deep inside the lumens of the various channels. It is vital to any decontamination process that these are cleaned and disinfected but ironically these are, in practice, difficult to sample. The danger with sampling more accessible surfaces is that this appears to give reassurance of a level of cleanliness throughout the whole scope that it does not in fact offer. Thus instead of staff working to a cleaning method that can effectively decontaminate the channels, they will work to a method that ensures that their test for cleaning passes and so may not put sufficient effort into cleaning those parts that they cannot sample.

Some United Kingdom (UK) and European endoscope reprocessing references available on the internet are given below. They do not refer to the use of monitoring the scopes after cleaning but instead concentrate on effective methods, agreed by endoscope manufacturers, of cleaning and disinfecting these difficult, delicate instruments (i.e. process control rather than product control).

I have less fundamental disagreement with cleaning assessment of cleaned surgical instruments but even here the arguments are not clearcut. My view is also biased from the UK perspective in that our current local perceptions are largely directed by prions. These are a particular problem in the UK and, as they cannot

\section{Corresponding author}

Peter Hoffman, Laboratory of Healthcare-associated Infection, Health Protection Agency, UK 
be inactivated by steam sterilisation, controlling transmission via surgical instruments is mainly via removal by cleaning and prevention of contamination by using disposable instruments on high risk tissues in high risk patients.

There is little use of ATP-based methods in the UK and some use of ninhydrin for protein detection but this is currently viewed as insufficiently sensitive and other methods are under investigation. However, this approach relates to a problem that has far less significance for the rest of the world. Also, even in the UK, only a small proportion of instruments will be sampled as an occasional validation that the cleaning process parameters are correct. As with the approach to endoscopes the problem remains that here too the easiest surfaces to sample are also the easiest to clean. In contrast those areas that are difficult to sample (e.g. lumens, hinges and the less accessible areas of instruments such as reamers, files and drills) are also difficult to clean. I feel that, for countries without a significant prion problem, visual inspection of every washed instrument remains a valid approach. The value of sampling a selected few instruments remain in need of interpretation.
There is further possible confusion in this area about why a particular result has been chosen as the dividing line between "clean" and "dirty" and what significance this has. However there is a similar indecisive division between a visual assessment of these parameters. There are no meaningful data to base either on in terms of infection control.

Given that ATP-based technology is relatively expensive for a large part of the world and that its use may divert people's attention from cleaning areas on endoscopes and instruments that cannot be sampled, I think that providing staff with the equipment and facilities they need as well as training and supervision is an answer with more direct relevance to infection control.

\section{Further reading}

1. British Society for Gasteroenterology endoscope decontamination guidelines 2005 can be downloaded at: http:// www.bsg.org.uk/pdf_word_docs/disinfection06.doc

2. European Society for Gastroenterology decontamination guidelines can be downloaded at http://www.esge.com/ assets/downloads/pdfs/guidelines/en_s77bis83_2000.pdf

3. The English \& Welsh Department of Health "Top Ten Tips" on endoscope reprocessing can be found at http://www. dh.gov.uk/en/Publicationsandstatistics/Bulletins/ Chiefexecutivebulletin/DH_4120886 or access the website and search on "endoscopes" 